\title{
BOOK PRESENTATION
}

\section{"BIOMEDICAL MEMBRANES AND (BIO)ARTIFICIAL ORGANS, World Scientific/ Oxford press, ISBN 978-981-3221-75-8.}

\section{Stamatialis, Editor}

Bioartificial organs group, Department of Biomaterials Science and Technology, TechMed Institute, University of Twente, the Netherlands

This book focusses on the development of biomedical membranes and their application for (bio)artificial organs. It covers the state of art and main challenges for applying synthetic membranes in these organs and it highlights the importance of accomplishing an integration of engineering with biology and medicine to understand and manage the scientific, industrial, clinical and ethical aspects of these organs. The book consists of the following 11 chapters.

\section{Chapter 1- Controlled Drug Release Systems: Mechanisms and Kinetics}

M. Sanopoulou and K. G. Papadokostaki,

Institute of Nanoscience and Nanotechnology, National Center for Scientific Research "Demokritos", Athens, Greece

This chapter begins with a presentation of the basic mechanisms in polymer-based controlled release systems and then it focuses on systems where diffusion plays a dominant role in determining the kinetics of release. Matrix systems are described in more detail and examples of relevant medical formulations are given.

\section{Chapter 2- Membranes for Artificial Kidney}

J. Vienken,

University of Applied Sciences, Gießen, Germany

This chapter begins with the introduction of the clinical need: blood purification via an artificial kidney and then focuses on important aspects related to the materials used for membrane fabrication, mass transfer consideration and biocompatibility issues.

\section{Chapter 3- Advanced Blood Purification Therapies}

O. de Beek, I. Geremia, D. Pavlenko and D. Stamatialis,

(Bio)artificial organs group, Department of Biomaterials Science and Technology, TechMed Institute, University of Twente, The Netherlands

This chapter focuses the application of membranes in advanced artificial blood purification systems including filtration, adsorption and their combination. Experimental, as well as commercially available systems are discussed including continuous therapy via application of wearable artificial system. 


\section{Chapter 4- Membranes for Artificial Lung and Gas Exchange Applications \\ F. Wiese \\ Consultant, Starenstr. 100, D - 42389 Wuppertal, Germany}

This chapter firstly introduces the clinical need: the application of membranes for artificial lung and gas exchange systems and then focuses on important aspects related to the materials used for membrane, mass transfer consideration and module design.

\section{Chapter 5- Membranes for Bioartificial Kidney}

N. V. Chevtchik ${ }^{1}$, P. Caetano-Pinto ${ }^{2}$ R. Masereeuw ${ }^{2}$ and D. Stamatialis ${ }^{1}$

${ }^{1}$ Bioartificial organs group, Department of Biomaterials Science and Technology, MIRA Institute, University of Twente, The Netherlands

${ }^{2}$ Pharmacology division, Department of Pharmaceutical Sciences, Utrecht University, The Netherlands

In recent years, the research around bioartificial kidneys has brought together different disciplines, combining technical expertise with cellular and molecular biology. This research is focused on the creation of devices that can mimic (partially) the function of a healthy kidney by combining of polymeric membranes and renal proximal tubule cells. This chapter focusses on the role of artificial polymeric membranes for the development of these devices.

\section{Chapter 6- Membrane-Based Bioartificial Liver Devices}

S. Khakpour 1,2, H. M. M. Ahmed ${ }^{1,2,}$ and L. De Bartolo ${ }^{1}$,

${ }^{1}$ Institute on Membrane Technology, National Research Council of Italy, Rende, Italy, ${ }^{2}$ Department of Chemical Engineering and Materials (DIATIC), University of Calabria, Rende, Italy.

Liver disease and the subsequent loss of liver function is an enormous clinical challenge. Since organ shortage remains a major hurdle, many researchers have developed various extracorporeal biohybrid artificial liver (BAL) systems which consist of functional liver cells supported by an artificial cell culture material. This chapter reviews the current state of the art related ot the membrane-based BAL which could potentially act as a bridge for the patients until a donor organ is available for transplantation.

\section{Chapter 7 - Are Co-culture Approaches Able to Improve Biological Functions of Membrane-based Bioartificial Livers?}

V. Pandolfi, U. Pereira, M. Dufresne and C. Legallais, Université de technologie de Compiègne, Sorbonne Universités, Compiègne, France

This chapter presents the most promising strategies for achieving translational BAL application, including changes of the cell environment, providing a three-dimensional configuration to the cells via the introduction of extracellular matrix (ECM) components and/or the establishment of selfassembled aggregates (e.g. spheroids), as well as the co-culture of various cell types. 


\section{Chapter 8 - Membranes for Bioartificial Pancreas}

K. Skryzpek ${ }^{1}$, A. van Apelddorn ${ }^{2}$ and D. Stamatialis ${ }^{1,}$

${ }^{1}$ (Bio)artificial organs group, Department of Biomaterials Science and Technology, University of Twente, The Netherlands

${ }^{2}$ Developmental bioengineering Department, Institute for Biomedical Technology, University of

Twente, The Netherlands

Clinical islet transplantation, although successful, is associated with several complications hindering the transplantation outcomes. The development of a bioartificial pancreas based on pancreatic cell encapsulation seems to be a promising strategy to overcome the issues related to intrahepatic transplantation. This chapter describes the development of membrane based macroencapsulation devices.

Chapter 9- Health economic evaluation during the translation of medical technologies: involving users in the design of bio-artificial pancreas transplantation scenarios for type I diabetes M. IJzerman ${ }^{1}, T$. Wissing ${ }^{1,2}$ and E. de Koning ${ }^{3}$

${ }^{1}$ University of Twente, MIRA institute for Biomedical Engineering and Technical Medicine, dept Health Technology \& Services Research, Enschede, The Netherlands

${ }^{2}$ Technical University Eindhoven, Faculty Biomedical Technology, dept. Soft Tissue Biomechanics and Tissue Engineering, Eindhoven, The Netherlands

${ }^{3}$ Leiden University Medical Center, Department of Medicine, Leiden, The Netherlands

This chapter introduces "early Health Technology Assessment" as a new paradigm in health economics to evaluate medical technologies in early stages of development and introduces Multi-Criteria Decision Analysis (MCDA) as one specific approach to support decisions in the development stage of new medical technologies. MCDA is illustrated using a case of the transplantation of a bioartificial pancreas (BAP) device.

\section{Chapter 10-Membranes for regenerative medicine in clinical applications}

\section{G. Falvo, D. Labate and G. Catapano}

Department of Environmental and Chemical Engineering, University of Calabria, Rende, Italy.

This chapter describes the application of membranes in regenerative medicine including their role as interfaces and scaffolds for cell culture as well as their role in proving specific transport and separation properties. The complex requirements specific to clinical treatments often stimulates combination of membranes with other biomaterials and adhesives, or introduction of post-processing techniques, to overcome the intrinsic limitations of available membranes.

\section{Chapter 11 - Membranes for organs on chips}

M. P. Tibbe ${ }^{1}$, L. I. Segerink ${ }^{1}$, A. D. van der Meer ${ }^{2}$, D. Stamatialis ${ }^{3}$ and A. van den Berg ${ }^{1}$

${ }^{1}$ BIOS Lab-on-a-chip group, MIRA \& MESA+Institutes, University of Twente, The Netherlands

${ }^{2}$ Applied Stem Cell Technologies group, MIRA institute, University of Twente, The Netherlands

${ }^{3}$ Bioartificial organs group, Department of Biomaterials Science and Technology, MIRA Institute, University of Twente, The Netherlands.

This chapter describes the role of membranes in the rapidly emerging field of organs on-chips (OOC), the reasons why specific membranes are used and where there are opportunities for improvement. Detailed presentation of the membrane materials and their properties relevant to OOC is discussed. 
